\title{
Seroprevalencia de la enfermedad de Chagas en Ushuaia, Argentina, una zona sin triatominos ${ }^{1}$
}

\author{
María Cristina Mallimaci, ${ }^{2}$ Carina Sijvarger ${ }^{2}$ Alejandro Dates, ${ }^{3}$ \\ Marcela Álvarez ${ }^{2}$ y Sergio Sosa-Estani ${ }^{4}$
}

RESUMEN Objetivos. Determinar la seroprevalencia de la infección por Trypanosoma cruzi en Ushuaia, la ciudad más austral del mundo.

Métodos. Se analizaron muestras de suero de 2991 personas, obtenidas entre enero de 1995 $y$ diciembre de 1996. Las muestras fueron procesadas por hemaglutinación indirecta (HAI) e inmunoensayo enzimático (ELISA) o inmunofluorescencia indirecta (IFI).

Resultados. La seroprevalencia general de la infección por T. cruzi fue de 6,8\%. La prevalencia según el país de origen fue de 41,1\% en los bolivianos, 5,0\% en los argentinos y 0,9\% en los chilenos; en embarazadas fue de 5,9\%, en exámenes obligatorios de $6,3 \%$ y en consultas dirigidas de 30,8\%.

Conclusiones. Se destaca la magnitud de la infección por T. cruzi en una zona donde no existe el insecto vector. Debido al riesgo de la transmisión congénita y transfusional, es necesario mantener el control de la sangre a transfundir y reforzar el seguimiento de los hijos de mujeres infectadas para un diagnóstico precoz y tratamiento oportuno de la infección.

Palabras clave Trypanosoma cruzi, enfermedad de Chagas, estudios seroepidemiológicos, Argentina.

En este trabajo se describe la seroprevalencia de la infección por Trypanosoma cruzi en las 2991 personas que

1 Presentado como comunicación en el V Congreso Argentino de Protozoología y Enfermedades Parasitarias, Sierra de la Ventana, 1997.

2 Servicio de Laboratorio, Hospital Regional Ushuaia. La correspondencia debe ser enviada a María Cristina Mallimaci, a la siguiente dirección: Hospital Regional Ushuaia, 12 de octubre y Maipú, 9410 Ushuaia, Tierra del Fuego, Argentina. Tel: 542901- 423200/422950. Fax: 54-2901- 421173. Correo electrónico: cmallimaci@infovia.com.ar

3 Servicio de Hemoterapia, Hospital Regional Ushuaia.

4 Servicio de Epidemiología, Centro Nacional de Diagnóstico e Investigación de Endemoepidemias (CeNDIE), ANLIS “Dr. Carlos G. Malbrán”. acudieron al Hospital Regional de Ushuaia, Provincia de Tierra del Fuego, extremo austral de América (figura 1), entre enero de 1995 y diciembre de 1996 para detectar reactividad contra T. cruzi por controles de embarazo, consultas externas y exámenes laborales o de radicación.

Las muestras se analizaron por hemaglutinación indirecta (HAI) (1) e inmunoensayo enzimático (ELISA) (2) o inmunofluorescencia indirecta (IFI) (3), con control de calidad (4), y se consideraron positivas cuando fueron reactivas al menos en dos pruebas (5).
La seroprevalencia global fue de $6,8 \%$. La mayor seroprevalencia correspondió a las personas nacidas en Bolivia $(41,1 \%)$, cifra similar a la registrada en algunos departamentos de ese país (6) y significativamente superior a la observada en personas provenientes de Argentina, Chile, otros países o de origen desconocido $\left(\chi^{2}=355,2 ; \mathrm{gl}=4\right.$; $P<0,001)$ (cuadro 1). Entre los argentinos la prevalencia fue diferente según la región de origen $\left(\chi^{2}=119,9 ; \mathrm{gl}=5\right.$; $P<0,001$ ), con cifras de $14,3 \%$ y $11,4 \%$ para las regiones del Noroeste y Nordeste, respectivamente, que constitu- 
CUADRO 1. Seroprevalencia de la infección por Trypanosoma cruzi según el país de nacimiento. Hospital Regional de Ushuaia, Tierra del Fuego, Argentina, 1995-1996

\begin{tabular}{lrrr}
\hline \multirow{2}{*}{$\begin{array}{c}\text { País de } \\
\text { nacimiento }\end{array}$} & $\begin{array}{c}\text { Total de } \\
\text { muestras }\end{array}$ & \multicolumn{2}{c}{ Reactivas } \\
\cline { 3 - 4 } & 1946 & No. & $\%$ \\
\hline Argentina & 175 & 72 & 41,1 \\
Bolivia & 224 & 2 & 0,9 \\
Chile & 51 & 1 & 2,0 \\
Otros & 595 & 30 & 5,0 \\
Desconocido & 2991 & 203 & 6,8 \\
$\quad$ Total & &
\end{tabular}

Diferencias: Argentina frente a Bolivia frente a Chile $\left(x^{2}=\right.$ 325,5; $\mathrm{gl}=2 ; P<0,001)$; Argentina frente a Bolivia: $\left(\chi^{2}=\right.$ $28,3 ; \mathrm{gl}=1 ; P<0,001)$, y Argentina frente a Chile: $\left(\chi^{2}=7,7\right.$; $\mathrm{gl}=1 ; P=0,005)$.

tancia observar la elevada seroprevalencia en embarazadas, próxima al 9\% registrado en la mayoría de las provincias endémicas argentinas (10) y en otras no endémicas para la transmisión vectorial (11). La situación actual en Ushuaia es la que se espera alcanzar en todo el país por el avance de las acciones de control de la transmisión vectorial y transfusional por parte del Programa Nacional de Chagas en Argentina $(10,12)$, que situará a la transmisión congénita como la principal vía de transmisión. Los fenómenos migratorios desde zonas endémicas de Argentina y países limítrofes hacen que Ushuaia deba ser considerada también como área endémica para la transmisión no vectorial de la infección por $T$. cruzi. Se debe mantener la vigilancia en los bancos de sangre para prevenir el contagio transfusional, e intensificar

el estudio de las embarazadas y de sus yeron el $74 \%$ de los casos del país, porcentaje similar al descrito por otros autores (7). No se observaron sueros reactivos entre las personas nacidas en las tres provincias más australes (Chubut, Santa Cruz y Tierra del Fuego). La prevalencia de infección entre aquellos estudiados por sospecha clínica o antedecentes epidemiológicos fue significativamente mayor que en los controles por embarazo $(5,9 \%)$ y en los exámenes laborales y de radicación $(6,3 \%)\left(\chi^{2}=\right.$ 38,$7 ; \mathrm{gl}=2 ; P<0,001$ ) (cuadro 2).

A pesar de que es una zona libre de triatominos (8), en Tierra del Fuego hay un riesgo real de adquirir la infección por T. cruzi, una enfermedad tropical, a través de las vías no vectoria- les. La seroprevalencia general de la infección en la ciudad de Ushuaia fue similar a la tasa de infectados estimada para el país (9). Es de especial impor-

CUADRO 2. Seroprevalencia de la infección por Trypanosoma cruzi según el motivo del estudio serológico. Hospital Regional de Ushuaia, Tierra del Fuego, Argentina, 1995-1996

\begin{tabular}{lccc}
\hline & & \multicolumn{2}{c}{ Reactivas } \\
\cline { 3 - 4 } \multicolumn{1}{c}{ Motivo del estudio serológico } & Total de muestras & No. & $\%$ \\
\hline Embarazo & 1311 & 78 & 5,9 \\
Examen laboral o de radicación & 1229 & 77 & 6,3 \\
Sospecha clínica o antecedente epidemiológico & 39 & 12 & 30,8 \\
\hline
\end{tabular}

Diferencias: $\chi^{2}=38,7 ; \mathrm{gl}=2 ; P<0,001$. 
hijos para detectar precozmente la enfermedad de Chagas congénita, cuyo riesgo se aproxima, según estudios recientes, a 6,7\% (13), y así brindarles tratamiento oportuno $(14,15)$.
Agradecimientos. A Alberto González por la recopilación de los datos; a Marck Wolfgang y a Esteban Mel por el procesamiento de los mismos; a Martín Urtasun por su revisión crítica y compa- ginación de los resultados; a Carlos López por su aporte en la redacción, y a Elsa Segura, Estela Cura y Ana De Rissio por su colaboración en la ejecución de este trabajo.

\section{REFERENCIAS}

1. Cerisola JA, Álvarez M, Wynne de Martini GJ, Bonacci $\mathrm{H}$. La reacción de hemoaglutinación cualitativa para el diagnóstico de la enfermedad de Chagas. Bioquímica Clínica 1971; 5:94.

2. Voller A, Draper C, Bidwell DE, Bartlett A. Microplate enzyme-linked immunosorbent assay for Chagas' disease. Lancet 1975;1(7904): 426-428

3. Alvarez M, Cerisola JA, Rohwedder RW. Test de inmunofluorescencia para el diagnóstico de la enfermedad de Chagas. Bol Chil Parasitol 1968;23:4-8.

4. Cura EN, Segura EL. Quality assurance of the serologic diagnosis of Chagas' disease. Rev Panam Salud Publica 1998;3:242-248.

5. Argentina, Ministerio de Salud y Acción Social de la Nación. Instituto Nacional de Chagas "Dr. Mario Fatala Chabén". Normas para el diagnóstico de la infección chagásica. Resolución ministerial $\mathrm{N}^{\circ}$ 523. Buenos Aires: Ministerio de Salud y Acción Social de la Nación; 1997.

6. Carrasco R, Miguez H, Camacho C, Echalar L, Revollo S, Ampuero T, et al. Prevalence of Trypanosoma cruzi infection in blood banks of seven departments of Bolivia. Mem Inst Osvaldo Cruz 1990;85:69-73.
7. Segura EL, Cura EN, Sosa-Estani SA, Andrade J, Lansetti JC, De Rissio AM, et al. Long-term effects of a nationwide control program on the seropositivity for Trypanosoma cruzi infection in young men from Argentina. Am J Trop Med Hyg 2000;62:353-362.

8. Carcavallo RU, Martínez A. Entomoepidemiología de la República Argentina. Investigaciones Científicas de las Fuerzas Armadas Argentinas 1968;13:1-143.

9. Esquivel ML, Segura EL. Estimación del número de infectados chagásicos en la Argentina. [Carta]. Medicina (B Aires) 1994;54: 91-92.

10. Iniciativa de Salud del Cono Sur (Incosur). VII Reunión de la Comisión intergubernamental para la eliminación del Triatoma infestans y la interrupción de la transmisión de la tripanosomiasis americana por transfusión; 1998 Mar 24-26; Buenos Aires. Washington, D.C.: Organización Panamericana de la Salud; 1998 pp.1-78. (Documento OPS/HPC/HCT/98.114).

11. Arcavi M, Orfus G, Griemberg G. Incidencia de la infección chagásica en embarazadas y en recién nacidos en área no endémica. Medicina (B Aires) 1993;53:217-222.

12. Segura EL, Sosa Estani S, Esquivel ML, Gómez, A, Salomón OD y Grupo de desa- rrollo y aplicación operativa. Control de la transmisión del Trypanosoma cruzi en Argentina 1999. Medicina (B Aires) 1999;59(supl. 2): 91-96.

13. Blanco SB, Segura EL, Gürtler RE. El control de la transmisión congénita de Trypanosoma cruzi en la Argentina. Medicina (B Aires) 1999;59(supl 2):138-142.

14. Sosa Estani S, Segura EL. Tratamiento de la infección por Trypanosoma cruzi en fase indeterminada. Experiencia y normatización en la Argentina. Medicina (B Aires) 1999;59(supl. 2):166-170

15. De Andrade ALS, Zicker F, de Oliveira RM, Almeida e Silva S, Luquetti A, Travassos LR, et al. Randomised trial of efficacy of benznidazole in treatment of early Trypanosoma cruzi infection. Lancet 1996; 348(9039):1407-1413.

Manuscrito recibido el 31 de mayo de 2001. Aceptado para publicación, tras revisión, el 27 de diciembre de 2000.

ABSTRACT Objective. Determine the seroprevalence of Trypanosoma cruzi infection in Ushuaia, Argentina, which is the southernmost city in the world.

Methods. Serum samples were analyzed from 2991 people, obtained between

Seroprevalence of Chagas' disease in Ushuaia, Argentina, an area without triatomines January 1995 and December 1996. The samples were processed using indirect hemagglutination and either enzyme-linked immunosorbent assay or indirect immunofluorescence.

Results. The general seroprevalence of T. cruzi infection was 6.8\%. According to the residents' country of origin, prevalence was $41.1 \%$ among Bolivians, 5.0\% among Argentines, and $0.9 \%$ among Chileans. The prevalence found in pregnant women was $5.9 \%$; in compulsory examinations (such as for a job or for immigrants settling permanently in Argentina), it was 6.3\%; and in examinations done based on clinical or epidemiological reasons to suspect infection with Chagas' disease, it was $30.8 \%$.

Conclusions. There is an alarming level of T. cruzi infection in this region, where the insect vector does not even exist. Given the risk of transmission congenitally and from blood transfusions, controls must be maintained on donated blood. In addition, follow-up of children of infected women should be strengthened so that these children receive early diagnosis and timely treatment of the infection. 